\title{
Tetrahydroxystilbene glucoside isolated from Polygonum multiflorum Thunb. demonstrates osteoblast differentiation promoting activity
}

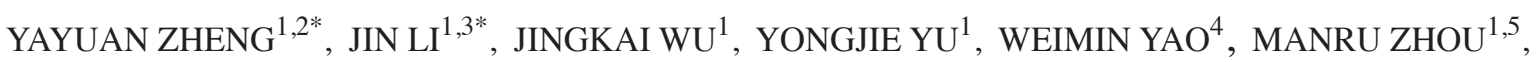 \\ JUN TIAN $^{6}$, JINGJING ZHANG $^{7}$, LIAO CUI ${ }^{1,8}$, XIAOBIN ZENG $^{8,9}$ and YUYU LIU ${ }^{1}$
}

\begin{abstract}
${ }^{1}$ Department of Pharmacology; ${ }^{2}$ Laboratory of Physiological Sciences, Guangdong Medical University, Zhanjiang, Guangdong 524023; ${ }^{3}$ Pharmacy Department, Heze Peony District Hospital of Traditional Chinese Medicine, Heze, Shandong 274009; ${ }^{4}$ Institute of Respiratory Disease, Affiliated Hospital of Guangdong Medical University, Zhanjiang, Guangdong 524023; ${ }^{5}$ Department of Pharmacy, Xinhua College of Sun Yat-Sen University, Guangzhou, Guangdong 510520; ${ }^{6}$ College of Life Science, Jiangsu Normal University, Xuzhou, Jiangsu 221116; ${ }^{7}$ Clinical Research Center, Affiliated Hospital of Guangdong Medical University; ${ }^{8}$ Guangdong Key Laboratory for Research and Development of Natural Drugs,

Department of Pharmacology, Guangdong Medical University, Zhanjiang, Guangdong 524023;

${ }^{9}$ Key Laboratory for New Drug Research of TCM and Shenzhen Branch, State R\&D Centre for Viro-Biotech, Research Institute of Tsinghua University in Shenzhen, Shenzhen, Guangdong 518057, P.R. China
\end{abstract}

Received April 5, 2016; Accepted April 21, 2017

DOI: $10.3892 / \mathrm{etm} .2017 .4915$

\begin{abstract}
Polygonum multiflorum Thunb. is a traditional Chinese medicinal herb that has been widely used to treat age-associated diseases. Tetrahydroxystilbene glucoside (TSG), also known as 2,3,5,4'-tetrahydroxystilbene-2-O- $\beta$-D-gluco side, is a major component of this herb. The present study was designed to investigate the osteogenic differentiation promoting activity of TSG in rat mesenchymal stem cells (MSCs) and in zebrafish. Preliminary experiments using MTT assay and ALP methods indicate that the high potential activity for promoting osteogenic differentiation was observed when $50 \%$ ethanol eluate was used. Further isolation and purification of TSG from the $50 \%$ ethanol eluate was performed by bioassay-guided fractionation, and its structure was confirmed using nuclear magnetic resonance and mass spectrometry analyses. In
\end{abstract}

Correspondence to: Professor Yuyu Liu, Department of Pharmacology, Guangdong Medical University, 2 Wenmingdong Road, Zhanjiang, Guangdong 524023, P.R. China

E-mail: liuyuyu77@163.com

Mr Xiaobin Zeng, Guangdong Key Laboratory for Research and Development of Natural Drugs, Department of Pharmacology, Guangdong Medical University, 2 Wenmingdong Road, Zhanjiang, Guangdong 524023, P.R. China

E-mail: 570778923@qq.com

*Contributed equally

Key words: tetrahydroxystilbene glucoside, Polygonum multiflorum Thunb. addition, the relative content of TSG with the highest potential activity in the promotion of osteogenic differentiation was identified as $14.34 \%$ by reversed-phase high performance liquid chromatography. Subsequently, the osteogenic differentiation promoting abilities of TSG in MSCs were examined. The results demonstrated that TSG promoted the alkaline phosphatase activity at concentrations of $1.56-25 \mu \mathrm{g} / \mathrm{ml}$, while it increased the content of osteocalcin 7 days after treatment with $6.25-25 \mu \mathrm{g} / \mathrm{ml}$ in MSCs. Furthermore, experiments in zebrafish indicated that different concentrations of TSG (3.12-12.5 $\mu \mathrm{g} / \mathrm{ml})$ protected against further bone loss induced by $10 \mu \mathrm{mol} / \mathrm{l}$ dexamethasone (Dex), simulating an osteoporosis (OP) model. TSG treatment $(12.5 \mu \mathrm{g} / \mathrm{ml})$ in Dex-induced zebrafish significantly increased the area of nodules by $50.14 \%$ compared with the untreated model group. In conclusion, TSG, as a major component of $P$. multiflorum Thunb. exhibited an osteogenic promoting activity in MSCs and in zebrafish. The results provided scientific evidence to support the potential use of TSG for protecting the bone in degenerative diseases, such as OP.

\section{Introduction}

Osteoporosis (OP) is a common multi-factorial bone disease associated with reduced bone mineral density, disordered bone microstructure architecture and increased fragility. OP may significantly affect the public health, thus resulting in a considerable cost for the economy and in increased morbidity and mortality (1). It is estimated that $>50 \%$ of adults aged $>50$ years suffer from OP, among which $70 \%$ are women with postmenopausal OP (2), while 9 million new osteoporotic fractures are estimated to occur globally each year (3). The primary cause of OP is bone metabolism disorders, and bone integrity requires a tight coupling between the activity of bone-forming 
osteoblasts and bone-resorbing osteoclasts (4). Bone formation is characterized by the proliferation and osteogenic differentiation of mesenchymal stem cells (MSCs). Alkaline phosphatase (ALP) is considered to be a marker of osteoblast differentiation that induced at early stages of bone formation, while osteocalcin (OCN) is detected at later stages (5).

Zebrafish, a teleost, are similar to mammals in terms of their genes. The external fertilization, speed of development, optical clarity, small size and fecundity of zebrafish, as well as the high homology compared with the human genome, have made them a prevalent vertebrate model in developmental biology investigations and as an animal model to study disease processes (6). With the continuous development and improvement of research methods, zebrafish bone as a novel model in vivo have provided a strong support for development research and protection of bone. Studies have demonstrated that zebrafish embryos or juveniles are a more complete system and contain the cells required for bone resorption and bone formation $(7,8)$. They have a broad prospect in chemical screening and selection of active ingredients such as traditional Chinese medicine and its active ingredients (9). Therefore, zebrafish were used in the present study to establish an animal OP model.

P. multiflorum Thunb. is a traditional Chinese medicinal herb that has been widely used for thousands of years in China, particularly to treat age-associated diseases (10). It has been demonstrated that male rats with OP treated with $P$. multiflorum Thunb. presented improved kidney 1-hydroxylase activity, bone mass, bone density, as well as maximum load of the lumbar region and femur (11). Previous studies have mainly focused on the crude extracts of P. multiflorum Thunb.; however, its chemical compounds with the anti-OP activity and the mechanism remain unclear at present. Tetrahydroxystilbene glucoside (TSG), also known as $2,3,5,4$ '-tetrahydroxystilbene-2-O- $\beta$-D-glucoside, is one of the major bioactive compounds in $P$. multiflorum Thunb. (12). Studies have focused on the various pharmacologic activities of TSG, including its anti-inflammatory (13), neurotoxicity protective (14), cardiotoxicity protective (15), anticancer (16) and anti-cardiovascular disorder (17) activities. However, the bioactivity of TSG on OP prevention has been rarely examined, with only one related paper showing that TSG can significantly enhance the osteogenic function in MC3T3-E1 cells by alleviating oxidative stress (18).

In the present study, the anti-osteoporotic compounds from the $P$. multiflorum Thunb. were isolated. TSG was isolated as a major component of this herb, using the part with the highest potential activity for promoting osteogenic differentiation (50\% ethanol eluate) by bioassay-guided fractionation (19) and identified on the basis of nuclear magnetic resonance (NMR) and mass spectrometry (MS) analyses. The relative content of TSG in the $50 \%$ ethanol eluate was determined and the potential osteogenic effects of the $50 \%$ ethanol eluate were evaluated in mesenchymal stem cells (MSCs). In order to clarify the effects and mechanism of TSG on osteogenic differentiation, the present study investigated the proliferation, differentiation and $\mathrm{OCN}$ content in rat MSCs. Furthermore, a dexamethasone (Dex)-induced OP model in zebrafish was used to investigate the anti-osteoporotic activity of TSG. In brief, the current study verified the major active constituent of $P$. multiflorum Thunb. (namely TSG) and demonstrated its anti-OP activity in MSCs and a zebrafish model.

\section{Materials and methods}

Materials and instruments. NMR spectra were recorded on a Bruker DPX-400 spectrometer (400 MHz for ${ }^{1} \mathrm{H}$ NMR; Bruker, Karlsruhe, Germany) using standard Bruker pulse programs. Chemical shifts were presented as the $\delta$-value with respect to tetramethylsilane, which was used as an internal standard. Electrospray ionisation-MS (ESI-MS) data were obtained on an Agilent 1200 HPLC/6410B TripleQuad mass spectrometer (Agilent, Technologies, Inc., Bremen, Germany). Silica gel (Qingdao Ocean Chemical Co., Ltd., Qingdao, China) and octadecylsilanized (ODS) silica gel (Macherey-Nagel, Duren, Germany) were used for column chromatography.

RP-18 $\mathrm{F}_{254}(0.25$ mm; Merck KGaA, Darmstadt, Germany) glass plates, and spots were visualized by spraying with $15 \% \mathrm{H}_{2} \mathrm{SO}_{4}$, followed by heating. High-performance liquid chromatography (HPLC) was performed using a Syncronis $\mathrm{C}_{18}$ column $(250 \times 4.6 \mathrm{~mm})$ and the Agilent 1200 mass spectrometry system. Sprague-Dawley rats were obtained from the Animal Center of Guangdong Medical College (Zhanjiang, China). Fetal bovine serum (FBS) and Dulbecco's modified Eagle's medium (DMEM) were purchased from Thermo Fisher Scientific, Inc. (Waltham, MA, USA). 3-(4,5-Dimethyl-2thiazolyl)-2,5-diphenyl-2-H-tetrazolium bromide (MTT), Para-Nitrophenylphosphate (PNPP), Dexamethasone (Dex), dimethyl sulfoxide (DMSO) and Alizarin red were purchased from Sigma-Aldrich; Merck KGaA. Epimedium flavonoid (EF) was obtained from the Chengdu Best Reagent Co., Ltd. (Chengdu, China). The ALP assay kit (cat. no. P0321) was purchased from the Beyotime Institute of Biotechnology (Jiangsu, China). An Osteocalcin ELISA kit (cat. no. CSB-E05129r) was purchased from Cusabio Biotech Co., Ltd. (Wuhan, China).

Plant material. P. multiflorum Thunb. was purchased in Henan, China. A voucher specimen was deposited at the herbarium of Guangdong Key Laboratory for Research and Development of Natural Drugs, Guangdong Medical College (Zhanjiang, China). The plant material was air-dried indoors at room temperature.

Extraction and isolation of herbal components. P. multiflorum Thunb. (10.0 kg) herb was extracted three times with $95 \%$ ethanol, followed by evaporation of the organic solvent under a vacuum at $55^{\circ} \mathrm{C}$, which yielded a crude extract of $1.80 \mathrm{~kg}$. Next, the crude extract was resuspended in water, added to a D101 macroporous resin column $(80 \times 1,200 \mathrm{~mm})$ and successively eluted with the following eluents (25 liters of each): $\mathrm{H}_{2} \mathrm{O}, 10 \%$ ethanol, $20 \%$ ethanol, $30 \%$ ethanol, $40 \%$ ethanol, $50 \%$ ethanol, $60 \%$ ethanol, $70 \%$ ethanol, $80 \%$ ethanol and then $90 \%$ ethanol. Each eluate was concentrated and dried under vacuum. MTT assay and ALP methods indicate that the highest potential activity for promoting osteogenic differentiation was observed when $50 \%$ ethanol eluate was used. Following this, further isolation and purification of TSG from the $50 \%$ ethanol eluent was performed as previously described (19). 

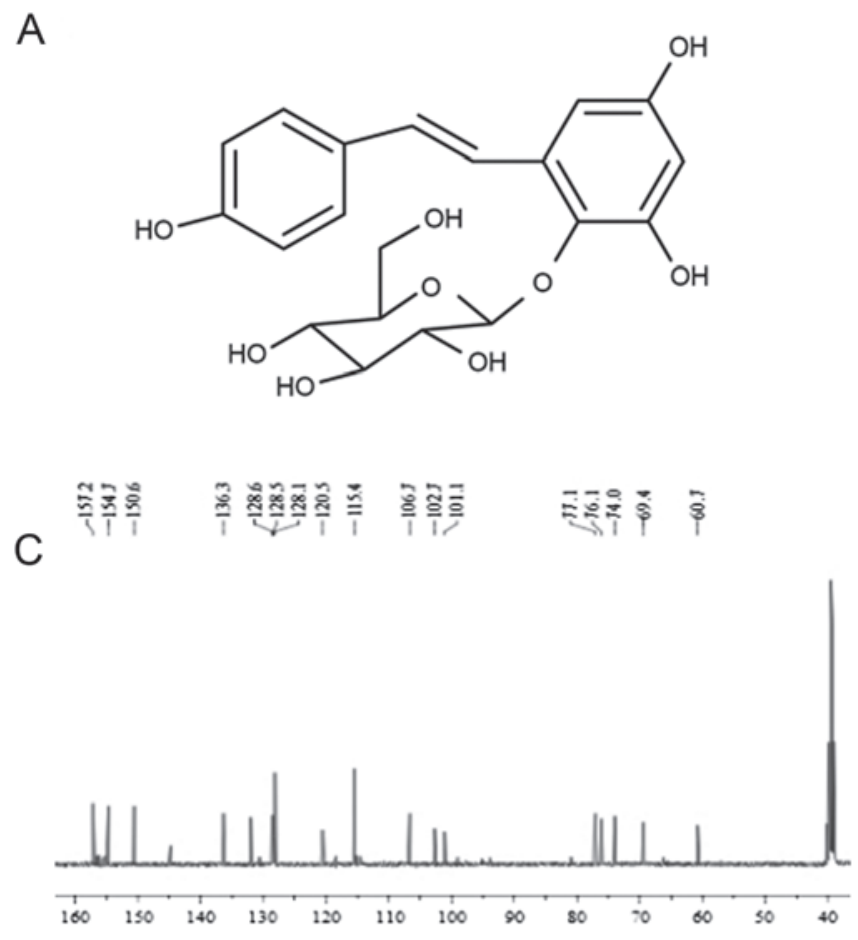

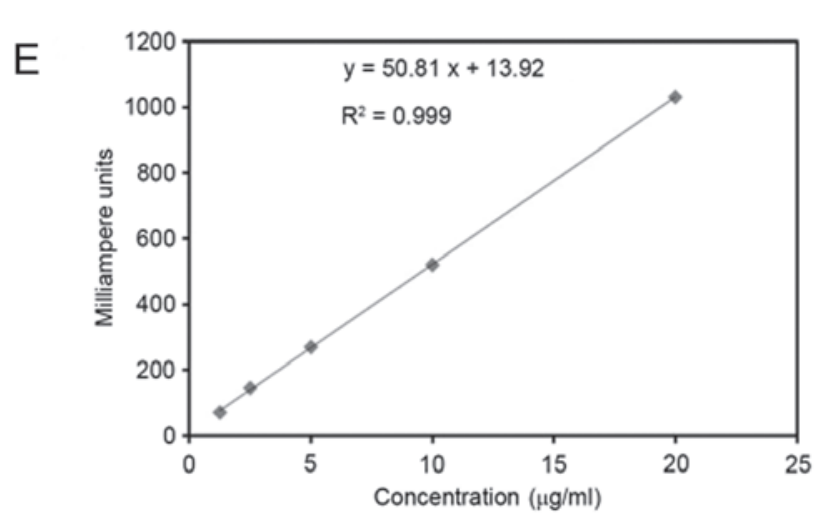

Figure 1. (A) Structure of TSG. (B) ${ }^{1} \mathrm{H}$ NMR analysis of TSG. (C) ${ }^{13} \mathrm{C}$ NMR analysis ofTSG. (D) High-performance liquid chromatography fingerprints of the $50 \%$ ethanol eluate over time. (E) Linearity of TSG calibration curve with regression equation. TSG, 2,3,5,4'-tetrahydroxy-stilbene-2-O- $\beta$-D-glucoside; NMR, nuclear magnetic resonance.

According to the bioactive preliminary results of the eluates, the $50 \%$ ethanol eluate presented the highest potential activity in promoting osteogenic differentiation. Therefore, the $50 \%$ ethanol eluate was fractionated over a silica gel (200-300 mesh) column, eluting with a gradually amount of $\mathrm{MeOH}$ in $\mathrm{CHCl}_{3}$ to obtain 10 fractions. The $\mathrm{CHCl}_{3}-\mathrm{MeOH}$ (7:3) eluate was further purified by silica gel column and Sephadex LH-20 together with preparative HPLC to obtain TSG after isolation and purification $(500.42 \mathrm{mg})$. The structure of TSG is shown in Fig. 1A.

HPLC analysis and quantitation of TSG in the 50\% ethanol eluate. HPLC was performed on a Syncronis $\mathrm{C}_{18}$ column $(250 \times 4.6 \mathrm{~mm})$ in Agilent 1200 to analyze the TSG in the $50 \%$ ethanol eluate under following conditions: mobile phase, (A) $\mathrm{MeOH}$; (B) $\mathrm{H}_{2} \mathrm{O}$ with $0.1 \% \mathrm{CF}_{3} \mathrm{COOH}$. The elution program was as follows: Linear gradient from $10 \%$ A to $30 \%$ $\mathrm{A}$ in $20 \mathrm{~min}, 30 \% \mathrm{~A}$ to $50 \% \mathrm{~A}$ in $20 \mathrm{~min}, 50 \% \mathrm{~A}$ to $85 \% \mathrm{~A}$ in
$20 \mathrm{~min}, 85 \%$ A to $100 \% \mathrm{~A}$ in $20 \mathrm{~min}$ and $100 \%$ A maintained for $20 \mathrm{~min}$. In addition, the following conditions were used: Detection wavelength, $300 \mathrm{~nm}$; flow rate, $0.80 \mathrm{ml} / \mathrm{min}$; injection volume, $10 \mu \mathrm{l}$; and column temperature, $25^{\circ} \mathrm{C}$.

The linearity of the calibration curve was determined by injecting TSG within the working range of the samples. Five serial dilutions, including 1.25, 2.50, 5.00, 10.00 and $20.00 \mu \mathrm{g} / \mathrm{ml}$, were prepared and injected in a total of $10 \mu \mathrm{l}$ dilutions in the HPLC column three times. The calibration graph was prepared by plotting the peak area against the corresponding TSG concentrations.

Cell culture of MSCs. All experiments were completed in compliance with the guidelines for animal care of the Medical Ethics Committee of the Guangdong Medical University. In order to examine the effects of TSG on osteogenic differentiation, MSCs were isolated from a total of 61 -month-old SD rats (weighing 100 $\pm 20 \mathrm{~g}, 50: 50$ male:female) obtained from 
the Experimental Animal Center of Guangdong Medical University (Foshan, China) by the whole bone marrow culture method (20), and the cell surface antigen was identified by flow cytometry. The rats were maintained at a temperature of $22-24^{\circ} \mathrm{C}$, humidity of $55 \pm 5 \%$, a 12 -h light/dark cycle and ad libitum access to food and water. Next, MSCs were cultivated in DMEM containing 10\% FBS, $100 \mu \mathrm{g} / \mathrm{ml}$ streptomycin and $100 \mathrm{U} / \mathrm{ml}$ penicillin at $37^{\circ} \mathrm{C}$ in a humidified incubator with $5 \% \mathrm{CO}_{2}$. Cells were seeded in $25 \mathrm{~cm}^{3}$ culture bottles at a density of $1 \times 10^{7}$ cells per $\mathrm{cm}^{2}$, and the medium was replaced every 3 days until the end of the culture period, when cells reached $80 \%$ confluence.

Cell proliferation assay. MSCs were seeded at a density of $5 \times 10^{3} / \mathrm{cm}^{2}$ cells per well in 96-well plates. After $24 \mathrm{~h}$, the MSCs were treated with culture medium containing different concentrations of $50 \%$ ethanol eluate $(1.56,3.12,6.25,12.5$, $25,50$ and $100 \mu \mathrm{g} / \mathrm{ml})$. After culturing for 24,48 and $72 \mathrm{~h}$, cell viability was examined using a standard MTT method (21).

ALP activity in MSCs. MSCs were seeded at a density of $1 \times 10^{4} / \mathrm{cm}^{2}$ cells per well in 48 -well plates. After $24 \mathrm{~h}$, the MSCs were treated with culture medium containing different concentrations of TSG $(1.56,3.12,6.25,12.5,25$ and $50 \mu \mathrm{g} / \mathrm{ml})$ or $50 \%$ ethanol eluate $(1.56,3.12,6.25,12.5,25,50,100$ and $200 \mu \mathrm{g} / \mathrm{ml}$ ), respectively to detect ALP. The concentration of TSG was determined according to the 50\% ethanol eluate concentrations. The ALP activity was determined using the ALP assay kit (Biotechnology, Jiangsu, China) (22). On day 3, 5, 7 and 9 days after treatment, cells were washed twice with $50 \mathrm{mM}$ phosphate-buffered saline (pH 7.4) and kept in $0.1 \%$ Triton X-100 lysis buffer overnight at $-20^{\circ} \mathrm{C}$. The cells were then thawed, and $300 \mu 1$ substrate buffer (containing $6.7 \mathrm{mmol} / 1$ disodium $p$-nitrophenylphosphate hexahydrate, $25 \mathrm{mmol} / \mathrm{l}$ diethanolamine and $1 \mathrm{mmol} / 1 \mathrm{MgCl}_{2}$ ) was added. After the mixtures were incubated at $37^{\circ} \mathrm{C}$ for $30 \mathrm{~min}$, the absorbance at $405 \mathrm{~nm}$.

OCN content analysis. MSCs were seeded at a density of $1 \times 10^{4} / \mathrm{cm}^{2}$ cells per well in 48 -well plates. After $24 \mathrm{~h}$, the MSCs were treated with culture medium containing different concentrations of TSG $(6.25,12.5$ and $25 \mu \mathrm{g} / \mathrm{ml})$ or $50 \mathrm{mg} / \mathrm{l}$ epimedium flavonoid (EF) for the positive control group. DMSO was used as a vehicle for the negative control group. The culture medium and drugs were replaced every 3 days. At 7 days after treatment, the conditioned media were collected for assessment. Subsequently, the content of OCN was measured according the standard curve of OCN. And the standard curve of OCN was measured following the instruction of the ELISA kit.

Zebrafish skeletal staining and quantification of bone mineralization. Zebrafish embryos were collected from mating of adult zebrafish, and reared under standard conditions, as previously described (23). Embryos were reared in embryo medium (containing $5 \mathrm{mM} \mathrm{NaCl}, 0.17 \mathrm{mM} \mathrm{KCl}$, $0.33 \mathrm{mM} \mathrm{CaCl}_{2}, 0.33 \mathrm{mM} \mathrm{Mg} \mathrm{SO}_{4}$ and $10^{-5} \%$ methylene blue) and staged for embryo development, using standard criteria (24). The zebrafish larvae at 4 days postfertilization (dpf) were transferred into 96-well plates containing embryo medium. Dex inhibits the production of bone morphogenetic protein 2 (BMP-2) in osteoblasts, which causes decreased MSCs differentiation into bone cells and was therefore used to induce a model of OP (25). Different concentrations of Dex $(20,10$ and $5 \mu \mathrm{g} / \mathrm{ml})$ or of TSG $(3.12,6.25$ and $12.50 \mu \mathrm{g} / \mathrm{ml})$ containing $10 \mu \mathrm{mol} / 1$ Dex were administered to 96 -well plates, with 12 fish per well, cultured for 5 days. The final volume of embryo medium per well was adjusted to $200 \mu 1$. Duplicate wells were set up for each concentration. Screening plates were incubated in the embryo incubator (IPP 400; Memmert $\mathrm{GmbH}+\mathrm{Co} . \mathrm{KG}$, Schwabacher, Germany) at $28.5 \pm 0.5^{\circ} \mathrm{C}$ until $9 \mathrm{dpf}$, at which point the zebrafish larvae were fixed in $4 \%$ paraformaldehyde solution at room temperature for $2 \mathrm{~h}$ and underwent skeletal staining with Alizarin red (Sigma-Aldrich; Merck KGaA) as described in a previous study (26). The head skeletons of Alizarin red-stained larvae were visualized and images were captured at the same threshold settings using a stereomicroscope with a Color Ciew Camera (Leica M205FA; Leica Microsystems, Wetzlar, Germany). Quantitative analysis was performed using Image Pro Plus version 6.0 (Media Cybernetics, Inc., Rockville, MD, USA) to determine the area of mineralized bones. The area of Alizarin red-stained larvae was quantified for each treatment group, with four samples per group.

Statistical analysis. All the quantitative data are presented as the mean \pm standard deviation. Statistical significance among groups was analyzed by the SPSS version 11.5 software (SPSS, Inc., Chicago, IL, USA) and evaluated using analysis of variance with Fisher's least significant difference test. Differences with P-values of $<0.05$ were considered as statistically significant.

\section{Results}

TSG identified from the extracts of P. multiflorum Thunb. TSG was isolated from the $50 \%$ ethanol eluate by bioassay-guided fractionation. TSG extracted from the herb was an amorphous yellow powder. The structure of TSG, namely 2,3,5,4'-tetrahydroxy-stilbene-2-O- $\beta$-D-glucoside, was identified using ${ }^{1} \mathrm{H}$ and ${ }^{13} \mathrm{C}$ NMR and ESI-MS (positive-ion mode). The ESI-MS spectra indicated an $[\mathrm{M}+\mathrm{Na}]^{+}$of $\mathrm{m} / z, 429$. In addition, the ${ }^{1} \mathrm{H}-\mathrm{NMR}$ spectra provided the following chemical shifts $\left(\delta_{\mathrm{H}}\right)$ : $6.19(1 \mathrm{H}, \mathrm{d}, J=2.8 \mathrm{~Hz}, \mathrm{H}-4), 6.56(1 \mathrm{H}, \mathrm{d}, J=2.8 \mathrm{~Hz}, \mathrm{H}-6), 7.68$ $(1 \mathrm{H}, \mathrm{d}, J=16.8 \mathrm{~Hz}, \mathrm{H}-7), 6.89(1 \mathrm{H}, \mathrm{d}, J=16.8 \mathrm{~Hz}, \mathrm{H}-8), 7.44$ $\left(1 \mathrm{H}, \mathrm{d}, J=8.4 \mathrm{~Hz}, \mathrm{H}-2^{\prime}\right), 6.74\left(1 \mathrm{H}, \mathrm{d}, J=8.4 \mathrm{~Hz}, \mathrm{H}-3^{\prime}\right), 6.74(1 \mathrm{H}$, d, $\left.J=8.4 \mathrm{~Hz}, \mathrm{H}-5^{\prime}\right), 7.44\left(1 \mathrm{H}, \mathrm{d}, J=8.4 \mathrm{~Hz}, \mathrm{H}-6^{\prime}\right), 4.41(1 \mathrm{H}, \mathrm{d}$, $\left.J=7.6 \mathrm{~Hz}, \mathrm{H}-1^{\prime \prime}\right), 3.69$ (1H, m, H-2"), 3.25 (1H, m, H-3"), 3.26 (1H, m, H-4"), 3.29 (1H, m, H-5"), 3.24 (1H, m, H-6"), 3.35 (1H, m, H-6"). The results observed in the ${ }^{13} \mathrm{C}-\mathrm{NMR}$ spectra $(100 \mathrm{MHz}$, DMSO- $\mathrm{d}_{6}$ ) were as follows: $\delta_{\mathrm{C}} 128.5(\mathrm{C}-1), 136.3(\mathrm{C}-2), 150.6$ (C-3), 101.1 (C-4), 154.7 (C-5), 106.7 (C-6), 120.5 (C-7), 132.0 (C-8), 128.6 (C-1'), 128.1 (C-2'), 115.4 (C-3'), $157.2\left(\mathrm{C}-4^{\prime}\right), 115.4$ (C-5'), 128.1 (C-6'), 102.7 (C-1"), 74.0 (C-2"), $77.1\left(\mathrm{C}-3^{\prime \prime}\right), 69.4$ (C-4"), $76.1(\mathrm{C}-5 "), 60.7$ (C-6"). The spectra of shifts is presented in Fig. 1B and C. And the spectral analysis was compared with literature data (27). The HPLC fingerprint of the 50\% ethanol eluate was then analyzed, as demonstrated in Fig. 1D. The result revealed that the peak area of TSG in the 50\% ethanol eluate was the largest. In order to determine the quantity of 


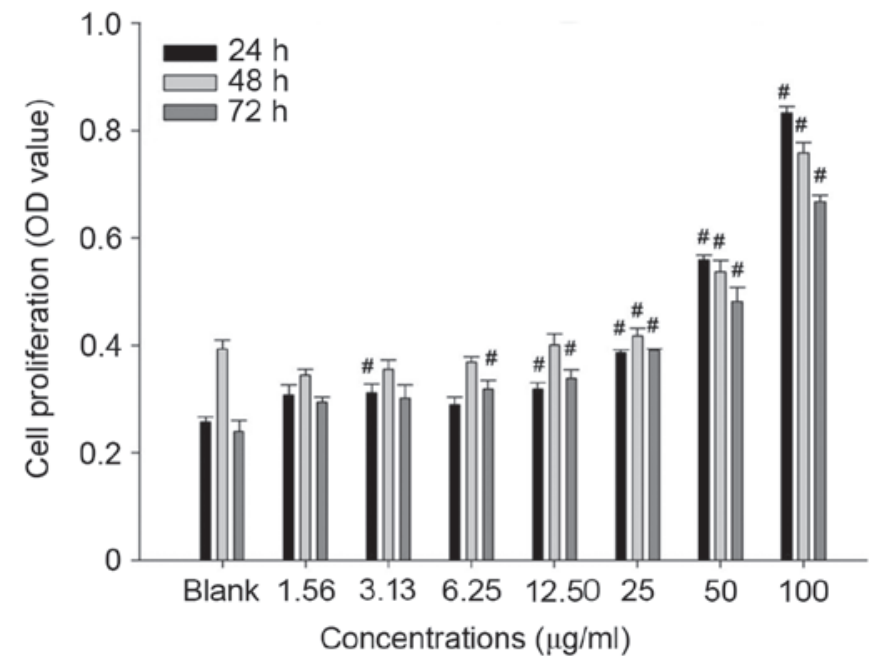

Figure 2. Effect of 50\% ethanol eluate on the proliferation of MSCs at different time points $(24,48$ and $72 \mathrm{~h}$ ) after treatment, as determined by MTT assay. ${ }^{~} \mathrm{P}<0.05$ vs. blank group. MSCs, mesenchymal stem cells; OD, optical density.

TSG in the $50 \%$ ethanol eluate, the method was performed by administering five concentrations of ethanol by injection, with concentrations ranging from 1.25 to $20 \mu \mathrm{g} / \mathrm{ml}$ (regression equation, $\mathrm{y}=50.81 \mathrm{x}+13.92$; coefficient of determination, $\mathrm{R}^{2} \geq 0.999$; Fig. 1E). The high correlation coefficients of the calibration curves of each concentration indicated good linearity among the range under investigation. The results revealed that the relative content of TSG in the $50 \%$ ethanol eluate was $14.34 \%$.

Effects of the $50 \%$ ethanol eluate on MSCs proliferation activity in vitro. To investigate the effects of the TSG containing $50 \%$ ethanol eluate on cell viability, an MTT assay was performed. The $50 \%$ ethanol eluate at concentrations of $12.5-100 \mu \mathrm{g} / \mathrm{ml}(\mathrm{P}<0.05)$ stimulated the MSCs proliferation in dose- and time-dependent manners. In addition, a significant increase in the proliferative activity was observed in cells treated with the $50 \%$ ethanol eluate after 24,48 and $72 \mathrm{~h}$ with a concentration $\geq 25 \mu \mathrm{g} / \mathrm{ml}(\mathrm{P}<0.05$; Fig. 2$)$, but $72 \mathrm{~h}$ may be limited due to space and the number of adherent cells counted may be less than the actual number. The $50 \%$ ethanol eluate at $100 \mu \mathrm{g} / \mathrm{ml}$ concentration demonstrated the highest promoting proliferative activity $(\sim 3.24$-fold) at $24 \mathrm{~h}$ after treatment, when compared with the blank group.

Effects of TSG on ALP activity of MSCs. The phenotype of mature osteoblasts is characterized by their ability to synthesize and secrete molecules of the extracellular matrix. One of the characteristics of a mature osteoblast phenotype is the ability of the cells to synthesize ALP, which is considered an early marker of osteogenic differentiation (28). Thus, in order to determine whether the $50 \%$ ethanol eluate and TSG stimulated osteogenic differentiation, the effects of the $50 \%$ ethanol eluate or TSG on ALP activity as a bone formation early marker were determined. The data illustrated that treatment of MSCs with the $50 \%$ ethanol eluate and TSG stimulated the ALP activity in a dose-dependent manner. As shown in Fig. 3A, Compared with the control, a concentration of $50-100 \mu \mathrm{g} / \mathrm{ml} \mathrm{50 \%}$ ethanol eluate significantly inhibited ALP
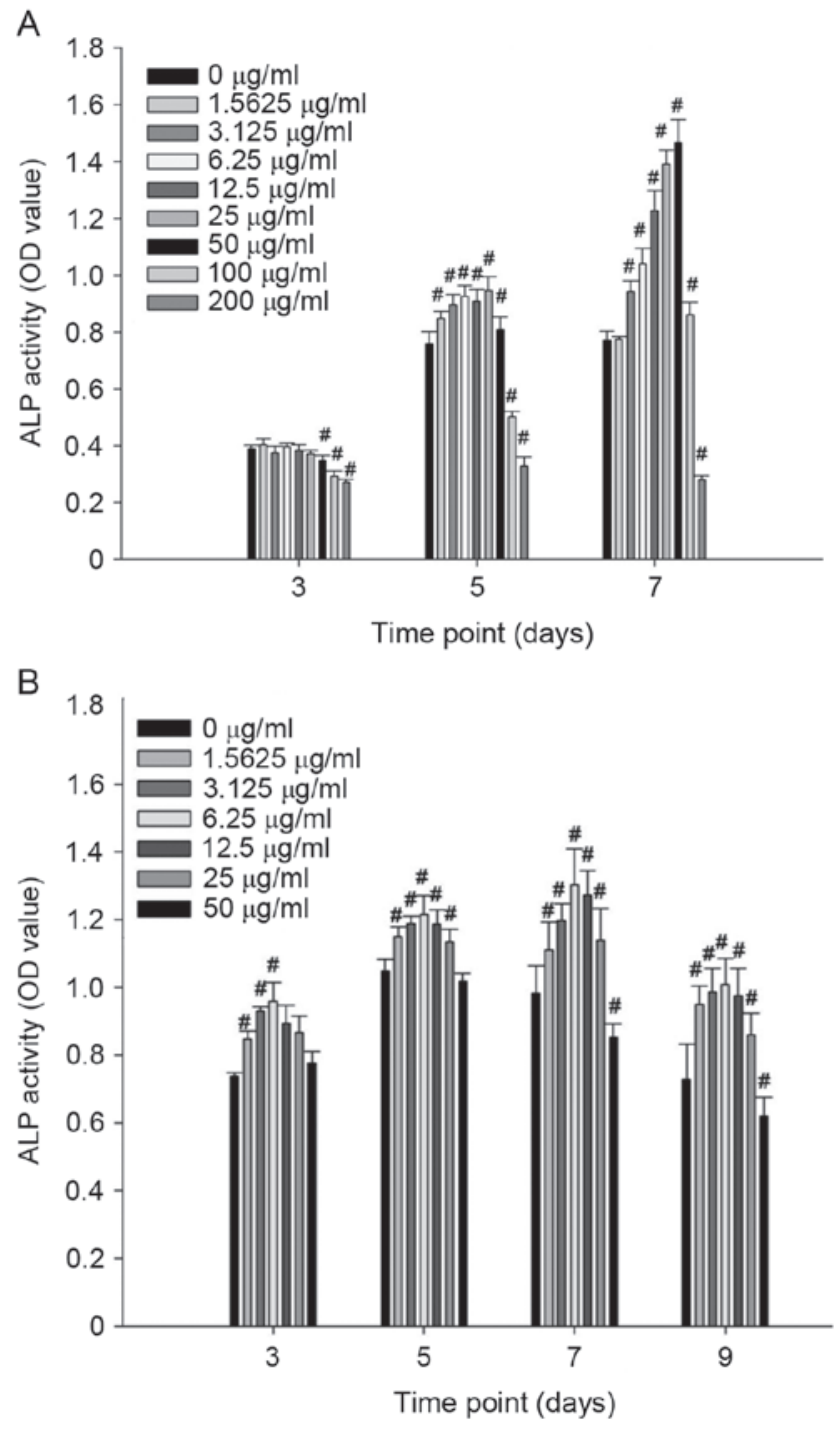

Figure 3. ALP activity was assessed in MSCs $(n=5)$ treated with (A) $50 \%$ ethanol eluate $(0-100 \mu \mathrm{g} / \mathrm{ml})$ for 3, 5 and 7 days, and (B) TSG $(0-50 \mu \mathrm{g} / \mathrm{ml})$ for $3,5,7$ and 9 days. ${ }^{~} \mathrm{P}<0.05$ vs. $0 \mu \mathrm{g} / \mathrm{ml}$ group. ALP, Alkaline phosphatase; MSCs, mesenchymal stem cells; TSG, tetrahydroxystilbene glucoside; OD, optical density.

expression on day 3 (Fig. 3A; P<0.05), while a the ALP expression significantly increased on day 5 days when treated with $1.56-50 \mu \mathrm{g} / \mathrm{ml}$ (Fig. 3A; $\mathrm{P}<0.05$ ), and was further increased on day 7 at a concentration of 3.12-50 $\mu \mathrm{g} / \mathrm{ml}$ (Fig. 3A; $\mathrm{P}<0.05$ ). As shown in Fig. 3B, ALP activity, following TSG treatment alone, was stimulated between 5 and 9 days $(\mathrm{P}<0.05)$, and reached a peak value on 7 days when treated with 1.56, 3.12, $6.25,12.5$ and $25 \mu \mathrm{g} / \mathrm{ml}$ TSG. By contrast, the expression of ALP was significantly inhibited when treated with $50 \mu \mathrm{g} / \mathrm{ml}$ TSG $(\mathrm{P}<0.05)$.

Effects of TSG on OCN content of MSCs. OCN is a non-collagenous protein found in the bone and dentin. OCN is secreted solely by osteoblasts, serves an important role in the body's metabolic regulation and is known to be pro-osteoblastic or bone-building. It is also implicated in bone mineralization and calcium ion homeostasis (29), and is often used as a marker for the bone formation process. Thus, the present study attempted to determine the effects of 

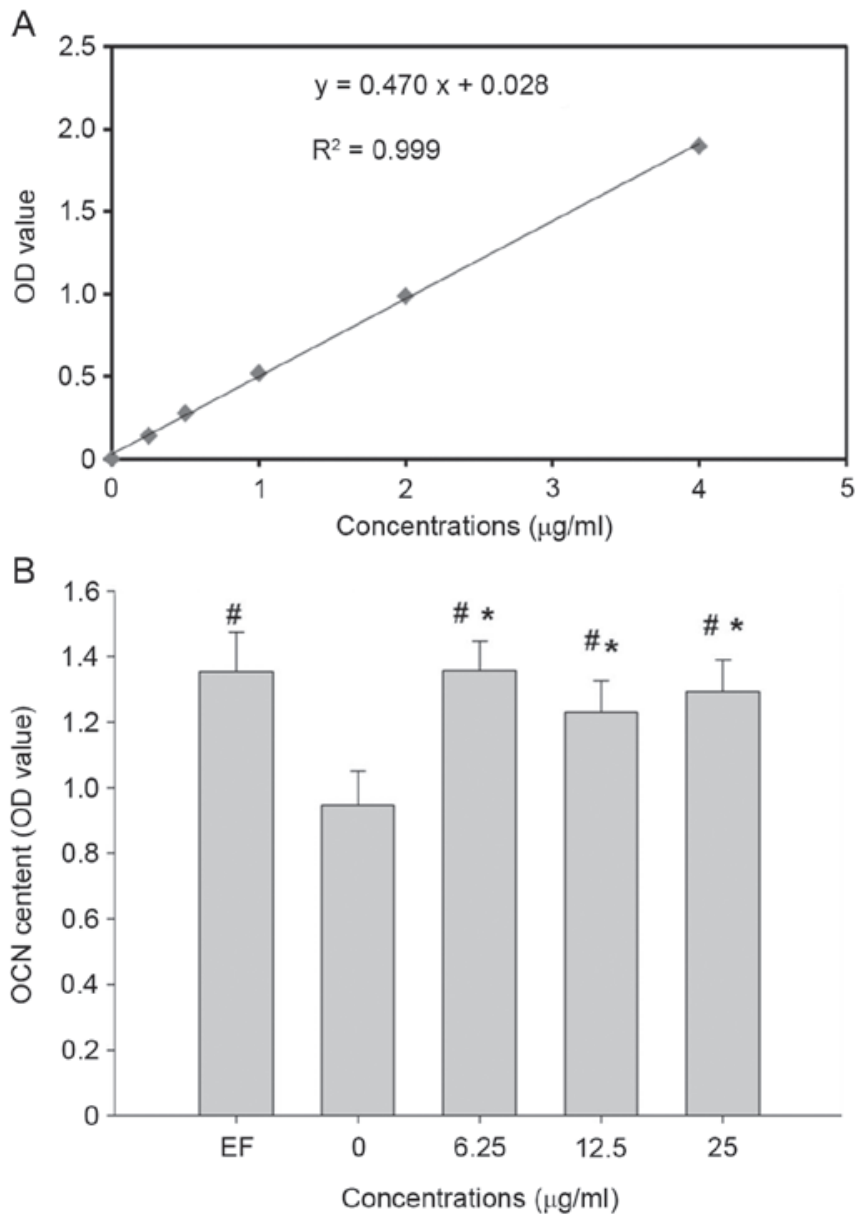

Figure 4. (A) Standard curve of the OCN content assessed using ELISA to calculate the expression of osteocalcin following treatment with EF or TSG over 7 days. (B) Effects of TSG on the OCN content were assessed in MSCs $(\mathrm{n}=6)$ treated with TSG $(0-25 \mu \mathrm{g} / \mathrm{ml})$ for 7 days. ${ }^{~} \mathrm{P}<0.05 \mathrm{vs} .0 \mu \mathrm{g} / \mathrm{ml}$ group; ${ }^{*} \mathrm{P}<0.05$ vs. $50 \mathrm{mg} / \mathrm{l} \mathrm{EF}$ group. OCN, osteocalcin; MSCs, mesenchymal stem cells; TSG, tetrahydroxystilbene glucoside; OD, optical density; EF, epimedium flavonoid.

TSG on the content of the bone formation marker OCN. As shown in Fig. 4A, an OCN standard curve was constructed $\left(\mathrm{R}^{2}>0.999\right)$ following instructions of the ELISA kit. Cells treated with EF for 7 days secreted increased OCN as compared with the control group (Fig. 4B). In addition, TSG treatment at concentrations of $6.25,12.5$ and $25 \mu \mathrm{g} / \mathrm{ml}$ increased the secretion of OCN in cells when compared with the control group $(\mathrm{P}<0.05$; Fig. 4B). A dose-dependent increase in OCN content by 43.37, 30.08 and $36.63 \%$ was observed for $6.25,12.5$ and $25 \mu \mathrm{g} / \mathrm{ml}$ TSG groups, as compared with the control (Fig. 4B).

TSG increases bone mineralization in larval zebrafish. The skeletal effects of TSG were detected in whole-mount skeletal preparations of zebrafish after 9 days of exposure. Compared with the vehicle-treated control, treatment with Dex at 5-20 $\mu \mathrm{mol} / 1$ caused an apparent decrease in the staining intensity and pixel number, which corresponds to the stained area (mineralized area), as shown in Fig. 5A. Using digital image analysis, the mineralized area was quantified for each sample in each treatment group. A clear dose-response correlation was observed, with the mineralized area decreasing with increasing doses of Dex (Fig. 5B). By contrast, an increase in mineralized area was observed in Dex-treated zebrafish following TSG treatment (Fig. 5C). Notably, $12.5 \mu \mathrm{g} / \mathrm{ml}$ TSG treatment in the $10 \mu \mathrm{mol} / 1$ Dex-treated zebrafish significantly increased the area of nodules by $\sim 50.14 \%$ compared with the DMSO-treated model group.

\section{Discussion}

Bone mass is maintained through a balance between osteoblastic bone formation and osteoclastic bone resorption (30). The imbalance caused by increasing bone resorption over bone formation leads to adult skeletal diseases, including OP, in which osteoblast development serves a key role.

Researchers have previously reported that compounds obtained from medicinal herbs are promising for the prevention and treatment of OP. P. multiflorum Thunb. a common Chinese medicinal herb, mainly contains three components: Stilbene glucosides $(\sim 1.0 \%)$, anthraquinone $(\sim 1.1 \%)$ and phospholipids $(\sim 3.7 \%)$ (31). It has been reported that TSG is the primary active substance in $P$. multiflorum Thunb. (13). In the present study, the MTT and ALP activity assays were conducted to determine the proliferative and osteogenic differentiation activity, respectively, of each ethanol eluate obtained from $P$. multiflorum Thunb. in vitro. The results demonstrated that the $50 \%$ ethanol eluate had the strongest effect on stimulating bone formation of MSCs at the tested concentrations (1.56-100 $\mu \mathrm{g} / \mathrm{ml})$. Subsequently, a major compound was isolated from the $50 \%$ ethanol eluate, and its structure was determined by ${ }^{1} \mathrm{H}$ NMR, ${ }^{13} \mathrm{C}$ NMR and ESI-MS. An RT-HPLC method was used to analyze the $50 \%$ ethanol eluate, and the results confirmed that TSG was the major constituent of the $50 \%$ ethanol eluate. In addition, the relative content of TSG in the $50 \%$ ethanol eluate was determined, which reached up to $14.34 \%$.

In order to determine whether the major compound (namely TSG) of the 50\% ethanol eluate was beneficial for the prevention and treatment of OP, the present study investigated its osteogenic stimulating activities in MSCs and zebrafish by examining various osteoblast differentiation markers (including ALP activity, OCN and bone mineralization). ALP, a cell membrane-associated enzyme, is a vital differentiation marker appeared in the early stages of differentiation and serves an important role on regulating the mineralization of the bone matrix (32). It is widely recognized as an early marker of osteogenic differentiation (33). In the current study, it was observed that MSCs treated with TSG at the tested concentrations $(1.56,3.12,6.25,12.5$ and $25 \mu \mathrm{g} / \mathrm{ml})$ significantly increased the ALP activity as compared with the control group. It has been demonstrated that differentiated osteoblasts also produce bone matrix, which is composed of collagenous proteins, mainly type I collagen, and non-collagenous proteins, such as OCN (34). OCN appears at a late stage of osteogenic differentiation and is involved in controlling the mineralization process (35). The results of the present study clearly indicated that TSG improved osteoblastic differentiation by promoting the levels of OCN. Thus, TSG is able to stimulate osteogenic differentiation of MSCs at both the initial and mature stages.

The increased expression of ALP and OCN following TSG treatment that was observed in the current study then contributes 
A Blank Vehicle $5 \mu \mathrm{mol} / 1 \mathrm{Dex}$ $10 \mu \mathrm{mol} / 1 \mathrm{Dex}$
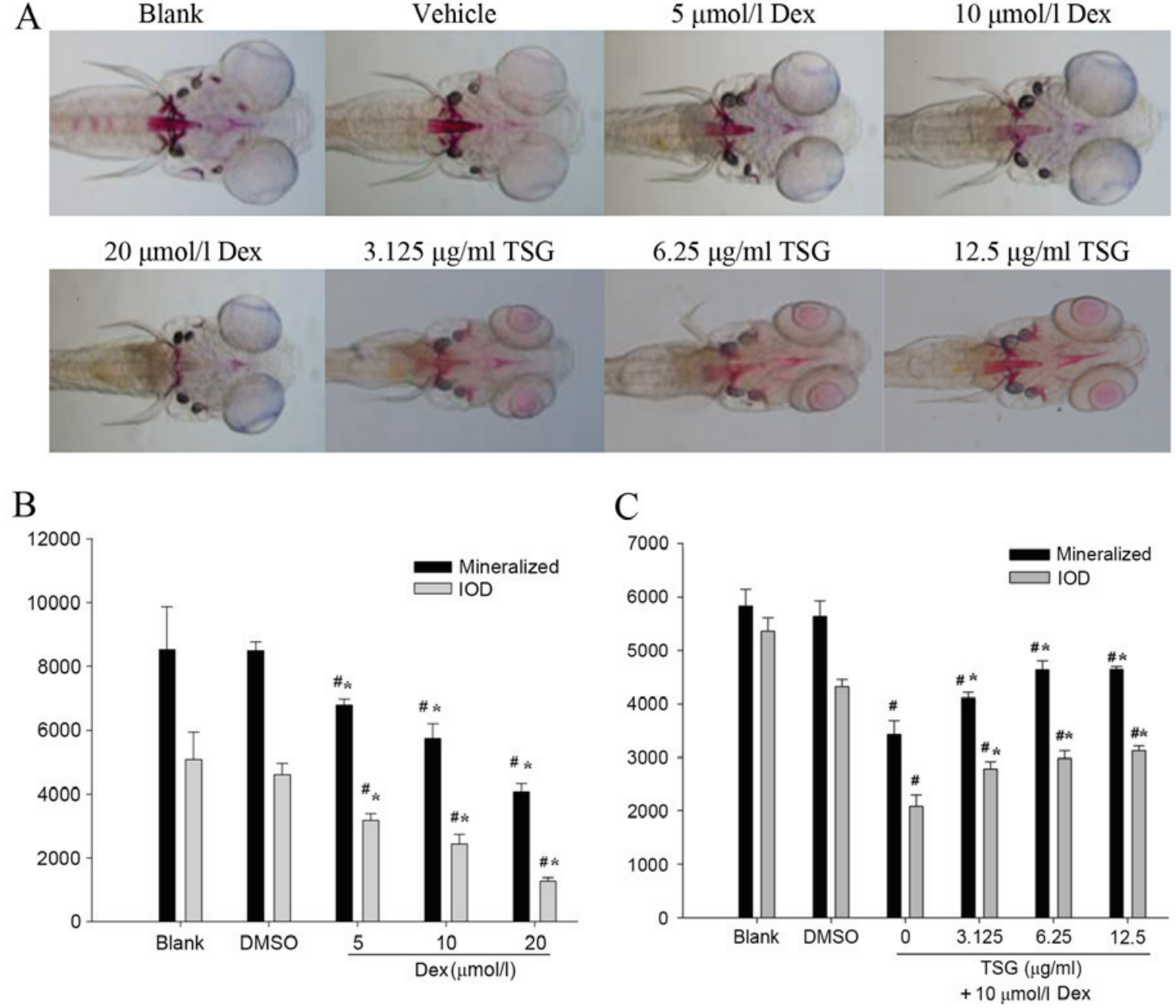

Figure 5. Effects of Dex and TSG on zebrafish larvae at 9 days post fertilization. (A) Ventral view of Alizarin red whole-mount preparations (magnification, x100). Areas of calcified matrix in craniofacial skeleton are stained red, while otoliths (not bony structures) appear brown/black. Compared with the blank and vehicle (0.5\% DMSO) control groups, the groups treated with 5, 10 and $20 \mu \mathrm{mol} / 1$ Dex demonstrated marked decrease in area and density of red staining. The TSG-treated groups $(3.125,6.25$ and $12.5 \mu \mathrm{g} / \mathrm{ml}$ ) presented significantly increased mineralized area and density of red staining. (B) Effects of Dex on mineralized area and IOD of mean pixel number of zebrafish larvae. " $\mathrm{P}<0.05$ vs. blank group; ${ }^{*} \mathrm{P}<0.05$ vs. DMSO control group. (C) Effects of TSG on mineralized area and IOD of mean pixel number of zebrafish larvae. "P $<0.05$ vs. DMSO control group; ${ }^{*} \mathrm{P}<0.05$ vs. $10 \mu \mathrm{mol} / 1 \mathrm{Dex}$ group. Dex, dexamethasone; TSG, tetrahydroxystilbene glucoside; IOD, integrated optical density; DMSO, dimethyl sulfoxide.

to the stimulation of osteoblastic differentiation. TSG also stimulated MSC differentiation and mineralization, which was consistent with the previous observation that TSG exerts a protective effect against hydrogen peroxide-induced dysfunction and oxidative stress in osteoblastic MC3T3-E1 cells (18).

Zebrafish has become a popular model of several human disease due to the scalability and potential vertebrate relevance of these animals (6). A zebrafish model is an established system for investigating developmental aspects of bone formation (36). In addition, the zebrafish genome in numerous cases has been demonstrated to contain a similar number of genes compared with the human genome, with high homology across key protein binding domains (6). For these reasons, a zebrafish model was used in the present study to assess whether TSG can stimulate bone formation in vivo. The current results revealed that the Dex exposure $(5,10$ and $20 \mu \mathrm{mol} / \mathrm{l})$ decreased the staining area and the staining optical density values of zebrafish head bones when compared with the vehicle control group (0.5\% DMSO), which suggested that Dex can significantly reduce the zebrafish mineralized bone and the bone mineral density. Treatment with
$6.25,12.5$ and $25 \mu \mathrm{g} / \mathrm{ml}$ TSG was found to significantly increase the mineralized matrix of the zebrafish head bone and prevent against osteopenia induced by $10 \mu \mathrm{mol} / 1 \mathrm{Dex}$. Therefore, TSG treatment of Dex-treated zebrafish was observed to prevent the Dex-induced osteopenia in vivo.

In conclusion, the present study was designed to isolate the anti-OP effective compound from P. multiflorum Thunb. MTT and ALP activity assays were used to examine the cell proliferation and osteogenic differentiation activity, respectively, of various ethanol eluates extracted from $P$. multiflorum Thunb. in vitro, with the $50 \%$ ethanol eluate demonstrating the strongest activity. TSG, a major component of this plant, was isolated and identified from the most active part, exhibiting the highest potential activity for promoting osteogenic differentiation (50\% ethanol eluate), and the relative content of TSG in the $50 \%$ ethanol eluate was calculated to be $14.34 \%$. To clarify the effects and mechanism of TSG on the osteogenic differentiation of MSCs in vitro, the cell proliferation, ALP activity and OCN content in MSCs were measured. In addition, a Dex-induced OP model in zebrafish was used to investigate the anti-OP 
activity of TSG in vivo. Taken together, the present manuscript verified that TSG, as a major constituent of P. multiflorum Thunb., demonstrated an anti-OP activity in vitro and in vivo. Furthermore, TSG may be the most important contributor for the anti-OP activity of P. multiflorum Thunb.

\section{Acknowledgements}

The present study was supported by grants from the National Natural Science Foundation of China (no. 81102450), the Guangdong Province Science and Technology Plan (no. 2012B031800225), the Characteristic Innovation Project (Natural Science) of the Education Department of Guangdong Province (no. 2014KTSCX084), the Science and Technology Innovation Fund of Guangdong Medical College (no. STIF201104) and the Open Project Fund of Guangdong Key Laboratory for Research and Development of Natural Drugs (no. 4CX16010G).

\section{References}

1. Woolf AD and Pfleger B: Burden of major musculoskeletal conditions. Bull World Health Organ 81: 646-656, 2003.

2. Simonelli $\mathrm{C}$ and Burke MS: Less frequent dosing of bisphosphonates in osteoporosis: Focus on ibandronate. Curr Med Res Opin 22: 1101-1108, 2006.

3. Johnell $\mathrm{O}$ and Kanis JA: An estimate of the worldwide prevalence and disability associated with osteoporotic fractures. Osteoporos Int 17: 1726-1733, 2006.

4. Rodan GA and Martin TJ: Therapeutic approaches to bone diseases. Science 289: 1508-1514, 2000.

5. Wang D, Christensen K, Chawla K, Xiao G, Krebsbach PH and Francesch RT: Isolation and characterization of MC3T3-E1 preosteoblast subclones with distinct in vitro and in vivo differentiation/mineralization potential. J Bone Miner Res 14: 893-903, 1999.

6. Goldsmith P: Zebrafish as a pharmacological tool: The how, why and when. Curr Opin Pharmacol 4: 504-512, 2004.

7. LeClair EE, Mui SR, Huang A, Topczewska JM and Topczewski J: Craniofacial skeletal defects of adult zebrafish Glypican 4 (knypek) mutants. Dev Dyn 238: 2550-2563, 2009.

8. Dimitriou R, Jones E, McGonagle D and Giannoudis PV: Bone regeneration: Current concepts and future directions. BMC Med 9: 66, 2011.

9. Peterson RT, Link BA, Dowling JE and Schreiber SL: Small molecule developmental screens reveal the logic and timing of vertebrate development. Proc Nat1 Acad Sci USA 97: 12965-12969, 2000.

10. Li X, Matsumoto K, Murakami Y, Tezuka Y, Wu Y and Kadota S: Neuroprotective effects of Polygonum multiflorum on nigrostriatal dopaminergic degeneration induced by paraquat and maneb in mice. Pharmacol Biochem Behav 82: 345-352, 2005.

11. Zhu FP, Wang HF and Gang LF: Effects of kidney-nourishing herbs on renal 1- $\alpha$-hydroxylase. Shanghai Zhong Yi Yao Za Zhi 37: 42-44, 2003 (In Chinese).

12. Liu C, Zhang Q and Zhou Q: Assay of stilbene glucoside in Polygonum multiflorum Thunb and its processed products. Zhongguo Zhong Yao Za Zhi 16: 469-472, 1991 (In Chinese).

13. Zhang YZ, Shen JF, Xu JY, Xiao JH and Wang JL: Inhibitory effects of 2,3,5,4'-tetrahydroxystilbene-2-O-beta-D-glucoside on experimental inflammation and cyclooxygenase 2 activity. J Asian Nat Prod Res 9: 355-363, 2007.

14. Qin R, Li XB, Li G, Tao LZ, Li Y, Sun J, Kang X and Chen J: Protection by tetrahydroxystilbene glucoside against neurotoxicity induced by $\mathrm{MPP}^{+}$: The involvement of PI3K/Akt pathway activation. Toxicol Lett 202: 1-7, 2011.

15. Zhang SH, Wang WQ and Wang JL: Protective effect of tetrahydroxystilbene glucoside on cardiotoxicity induced by doxorubicin in vitro and in vivo. Acta Pharmacol Sin 30: 1479-1487, 2009.
16. Jiang Z, Xu J, Long M, Tu Z, Yang G and He G: 2,3,5,4'-tetrahydroxystilbene-2-O-beta-D-glucoside (THSG) induces melanogenesis in B16 cells by MAP kinase activation and tyrosinase upregulation. Life Sci 85: 345-350, 2009.

17. Zhang W, Xu XL, Wang YQ, Wang CH and Zhu WZ: Effects of 2,3,4',5-tetrahydroxystilbene-2-O-beta-D-glucoside on vascular endothelial dysfunction in atherogenic-diet rats. Planta Med 75: 1209-1214, 2009

18. Zhang JK, Yang L, Meng GL, Fan J, Chen JZ, He QZ, Chen S, Fan JZ, Luo ZJ and Liu J: Protective effect of tetrahydroxystilbene glucoside against hydrogen peroxide-induced dysfunction and oxidative stress in osteoblastic MC3T3-E1 cells. Eur J Pharmacol 689: 31-37, 2012.

19. Zeng X, Tian J, Cai K, Wu X, Wang Y, Zheng Y, Su Y and Cui L: Promoting osteoblast differentiation by the flavanes from Huangshan Maofeng tea is linked to a reduction of oxidative stress. Phytomedicine 21: 217-224, 2014.

20. Zhang L and Chan C: Isolation and enrichment of rat mesenchymal stem cells (MSCs) and separation of single-colony derived MSCs. J Vis Exp: pii: 1852, 2010.

21. He X and Liu RH: Triterpenoids isolated from apple peels have potent antiproliferative activity and may be partially responsible for apple's anticancer activity. J Agric Food Chem 55: 4366-4370, 2007.

22. Rao LG, Liu LJ, Murray TM, McDermott E and Zhang X: Estrogen added intermittently, but not continuously, stimulates differentiation and bone formation in SaOS-2 cells. Biol Pharm Bull 26: 936-945, 2003.

23. Westerfield M: The Zebrafish Book. A Guide for the Laboratory Use of Zebrafish (Danio rerio). 2.1st edition. University of Oregon Press, Eugene, OR, 1994.

24. Kimmel CB, Ballard WW, Kimmel SR, Ullmann B and Schilling TF: Stages of embryonic development of the zebrafish. Dev Dyn 203: 253-310, 1995.

25. Zhang M, Sun FJ, Yu HJ, Lin Z, Sheng GL and Li GH: The effect of chilk extracts on dexamethasone-induced osteoporosis rat model. Chin J Osteoporosis 17: 589-592, 2011.

26. Kelly WL and Bryden MM: A modified differential stain for cartilage and bone in whole mount preparations of mammalian fetuses and small vertebrates. Stain Technol 58: 131-134, 1983.

27. Lv L, Shao X, Wang L, Huang D, Ho CT and Sang S: Stilbene glucoside from Polygonum multiflorum Thunb.: A novel natural inhibitor of advanced glycation end product formation by trapping of methylglyoxal. J Agric Food Chem 58: 2239-2245, 2010.

28. Orimo H: The mechanism of mineralization and the role of alkaline phosphatase in health and disease. J Nippon Med Sch 77: 4-12, 2010.

29. Lee NK, Sowa H, Hinoi E, Ferron M, Ahn JD, Confavreux C, Dacquin R, Mee PJ, McKee MD, Jung DY, et al: Endocrine regulation of energy metabolism by the skeleton. Cell 130: 456-469, 2007.

30. Seeman E and Delmas PD: Bone quality-the material and structural basis of bone strength and fragility. N Engl J Med 354: 2250-2261, 2006.

31. Chen S, Zou L and Wu T: Research progress of Polygonum multiflorum for preventing and treating osteoporosis. J Mod Med Health 27: 3428-3431, 2011

32. Hessle L, Johnson KA, Anderson HC, Narisawa S, Sali A, Goding JW, Terkeltaub R and Millan JL: Tissue-nonspecific alkaline phosphatase and plasma cell membrane glycoprotein-1 are central antagonistic regulators of bone mineralization. Proc Natl Acad Sci USA 99: 9445-9449, 2002.

33. Serigano K, Sakai D, Hiyama A, Tamura F, Tanaka M and Mochida J: Effect of cell number on mesenchymal stem cell transplantation in a canine disc degeneration model. J Orthop Res 28: 1267-1275, 2010.

34. Bao X, Liu Y, Han G, Zuo Z and Hu M: The effect on proliferation and differentiation of cementoblast by using sclerostin as inhibitor. Int J Mol Sci 14: 21140-21152, 2013.

35. Sun H, Feng K, Hu J, Soker S, Atala A and Ma PX: Osteogenic differentiation of human amniotic fluid-derived stem cells induced by bone morphogenetic protein-7 and enhanced by nanofibrous scaffolds. Biomaterials 31: 1133-1139, 2010.

36. Du SJ, Frenkel V, Kindschi G and Zohar Y: Visualizing normal and defective bone development in zebrafish embryos using the fluorescent chromophore calcein. Dev Biol 238: 239-246, 2001. 